\title{
Meeting the Challenge of Diabetes in China
}

\author{
Zhen Luo $^{1}{ }^{\mathbb{D}}$, Guilhem Fabre ${ }^{2}$, Victor G. Rodwin ${ }^{3^{*}}$
}

\begin{abstract}
China's estimated 114 million people with diabetes pose a massive challenge for China's health policy-makers who have significantly extended health insurance coverage over the past decade. What China is doing now, what it has achieved, and what remains to be done should be of interest to health policy-makers, worldwide. We identify the challenges posed by China's two principal strategies to tackle diabetes: (1) A short-term pilot strategy of health promotion, detection and control of chronic diseases in 265 national demonstration areas (NDAs); and (2) A long-term strategy to extend health promotion and strengthen primary care capacity and health system integration throughout China. Finally, we consider how Chinese innovations in artificial intelligence (AI) and Big Data may contribute to improving diagnosis, controlling complications and increasing access to care. Health system integration in China will require overcoming the fragmentation of a system that still places excessive reliance on local government financing. Moreover, what remains to be done resembles deeper challenges faced by healthcare systems worldwide: the need to upgrade primary care and reduce inequalities in access to health services.

Keywords: China, Diabetes, Health Policy, Artificial Intelligence, Big Data

Copyright: @ 2020 The Author(s); Published by Kerman University of Medical Sciences. This is an open-access article distributed under the terms of the Creative Commons Attribution License (http://creativecommons.org/ licenses/by/4.0), which permits unrestricted use, distribution, and reproduction in any medium, provided the original work is properly cited.

Citation: Luo Z, Fabre G, Rodwin VG. Meeting the challenge of diabetes in China. Int J Health Policy Manag. 2020;9(2):47-52. doi:10.15171/ijhpm.2019.80
\end{abstract}

\section{Article History:}

Received: 27 June 2019

Accepted: 18 September 2019

ePublished: 6 October 2019
*Correspondence to:

Victor G. Rodwin

Email:vgrodwin@gmail.com

\section{Introduction}

China accounts for the largest number of people with diabetes (114 million), ${ }^{1}$ mainly type 2 , within a single nation. This poses a massive challenge for China's policy-makers who have significantly extended health insurance coverage over the past decade. Medical management of diabetes, alone, before complications, is already estimated to account for $8.5 \%$ of national health expenditure in China. ${ }^{1-3}$ As Professor Linong Ji, Vice President of the International Diabetes Federation averred, if diabetes is not controlled in China, "the wealth that we've accumulated over the past 30 years will be wasted in the drains of our dialysis machines."

What China is now doing, what it has achieved, and what remains to be done, can be instructive for health policymakers, worldwide. Based on journal articles in Chinese, government sources and peer-reviewed research articles, in English, we review prevalence estimates, variations by geographic areas, and assess China's accomplishments and the two principal strategies it has implemented to date: (1) A short-term pilot strategy of health promotion, detection and control of chronic diseases in 265 national demonstration areas (NDAs); and (2) A long-term strategy to extend health promotion and strengthen primary care capacity and health system integration among local communities and health providers, including hospitals, throughout China. We also consider how Chinese innovations in artificial intelligence (AI) and Big Data may contribute to health promotion, detection and management of diabetes in the future.

\section{Diabetes Prevalence: National Average and Variations}

Prevalence estimates of disease are highly dependent on the assumptions, definitions and survey methods used, all of which require judgment calls and interpretation. Thus, it is not surprising that five recent surveys, in China, arrive at different estimates of diabetes prevalence (Table 1). Although these surveys were conducted at different times, the differences may also reflect different methods (including selection of areas and samples, sample size, blood glucose testing indicators, population age structure) rather than disease prevalence. For example, the 1994 survey $^{5}$ was not based on a nationally representative sample and the two most recent surveys (2010 and 2013) (6,7 $^{6}$ apply 2010 criteria from the American Diabetes Association's (ADA's) definition, while the 2007-2008 and 1994 surveys applied more restrictive criteria from the World Health Organization (WHO). ${ }^{5,8}$

Even after taking into account methodological differences among these surveys, the four-fold growth in diabetes prevalence since 1994 is striking (Table 1).5,7 The socioeconomic and cultural determinants of this growth are most often attributed to the transformation of diet, ${ }^{10}$ genetic factors, ${ }^{11}$ the effects of the Great Famine (1958-1962) (2,13 $^{13}$ and its intergenerational effects. ${ }^{13}$ In Chinese megacities, children are typically raised by their grandparents, most of whom experienced the famine. This experience has likely contributed to their high-calorie intake and low physical activity, and in this way exacerbated the intergenerational transmission of risk factors for diabetes. 
Table 1. National Surveys of Diabetes in China

\begin{tabular}{|c|c|c|c|c|}
\hline Year of the Survey & Prevalence Estimate (\%) & $\begin{array}{l}\text { Detection Rate (Patients Aware of Their } \\
\text { Condition) (\%) }\end{array}$ & $\begin{array}{c}\text { Prediabetes Prevalence } \\
\text { Estimate (\%) }\end{array}$ & Source \\
\hline $1994^{\mathrm{a}}$ & 2.51 & 29.6 & 3.23 & Pan et al, $1997^{5}$ \\
\hline $2000-2001^{b}$ & 5.49 & - & 7.33 & Hu et al, $2009^{9}$ \\
\hline $2007-2008^{c}$ & 9.7 & 39.3 & 15.5 & Yang et al, $2010^{8}$ \\
\hline $2010^{d}$ & 11.6 & 30.1 & 50.1 & Xu et al, $2013^{6}$ \\
\hline $2013^{e}$ & 10.9 & 36.5 & 35.7 & Wang et al, $2017^{7}$ \\
\hline
\end{tabular}

${ }^{\text {a }}$ A survey based on a sample of 250000 adults aged 25 years or older, in 19 areas, including cities and rural areas of the north, south, east, west, and middle part of China. Diagnostic criteria from WHO, 1985.

${ }^{\mathrm{b}}$ A survey based on a population of 19012 adults, aged 35-74 and randomly selected from 20 primary sampling units (street districts in urban areas, townships in rural areas) and invited to participate. Diagnostic criteria from ADA.

${ }^{c} A$ survey based on a nationally representative sample of 46239 adults, 20 years of age or older, from 14 provinces and municipalities participated in the study. Diagnostic criteria from WHO, 1999.

${ }^{\mathrm{d}} \mathrm{A}$ cross-sectional survey in a nationally representative sample of 98658 adults. Diagnostic criteria from the ADA, 2010.

${ }^{e} \mathrm{~A}$ nationally representative cross-sectional survey of 170287 adults; minority ethnic groups in China with at least 1000 participants (Tibetan, Zhuang, Manchu, Uyghur, and Muslim) were compared with Han participants. Diagnostic criteria from the ADA, 2010.

Another striking finding from the surveys is the low share of people with diabetes who are aware of their illness (the detection rate), a significant indicator since diabetes patients who do not control their blood sugar levels are likely to end up with cardiovascular disease or kidney failure. The most recent survey ${ }^{7}$ indicates a $36.5 \%$ detection rate in 2013, which is much lower than in France $(67.24 \%),{ }^{14}$ the United States $(75.65 \%),{ }^{15}$ the United Kingdom (83.55\%), ${ }^{16}$ Australia $(71 \%),{ }^{17}$ and India $(47.98 \%) .{ }^{18}$

\section{Geographic Variation}

There are enormous variations in diabetes prevalence among urban and rural areas and wealthy and poorer regions. In 2016, urban versus rural residents' annual income (33616 yuan) was almost 3 times that of their rural counterparts (12363 yuan). ${ }^{19}$ Based on the 2010 survey, prevalence of diabetes ranges from $14.3 \%$ in urban versus $10.3 \%$ in rural areas. ${ }^{6}$ Higher-income urban counties have a higher average prevalence rate $(13.1 \%)$, than rural low-income counties $(8.7 \%)^{20}$; or according to a 2013 survey, $12.6 \%$ in urban versus $9.5 \%$ in rural areas. ${ }^{7}$ Based on a 2010 survey, adults with diabetes living in rural areas are less likely to be aware of their illness (38.7\%) than their counterparts in urban areas $(24.6 \%)^{6}$; according to another 2010 survey, the detection rate ranges from $40.8 \%$ in higher-income urban counties to $20.5 \%$ in lower-income rural counties ${ }^{20}$; according to the 2013 survey, $43.1 \%$ in urban and $29.1 \%$ in rural areas. ${ }^{7}$ In general, people residing in wealthier urban areas are more likely to have diabetes. Among the poorest populations in these areas, however, due to a lower level of health awareness, they face lower odds of having their condition detected and managed. ${ }^{20}$

\section{Migrant Workers and Urban-Rural Variation}

China has experienced the world's most rapid urbanization over the past two decades. Attracted by higher wages, millions of rural residents have migrated to cities. Those who have left their hometown for more than 6 months and have not been able to register as official residents in cities are considered migrant workers. Although their number has declined from a peak of 253 million (in 2014), to 245 million migrants (in 2016), ${ }^{21}$ they still pose a public health challenge since most municipal governments regard them as outsiders and their health insurance benefits (from home provinces) are typically insufficient to assure them of access to healthcare in cities.

Although migrant workers face fewer risk factors for diabetes, since most healthy migrants participate in heavy labor, ${ }^{22}$ once they become diabetic, or even prediabetic, they are more likely to forgo medical care due to financial barriers than official urban residents. ${ }^{23}$ Since diabetes is associated with increased mortality from a range of complications, their health status is likely to deteriorate faster than that of their official urban counterparts. ${ }^{22}$ Given the low diabetes detection rates (Table 1) and the low treatment rates for those diagnosed with diabetes, there is evidence of excess mortality of diabetes patients, which points to inadequate health promotion and clinical management of diabetes. ${ }^{24,25}$

\section{National Strategies: Achievements and Problems}

In the Healthy China 2030 Plan, approved by the State Council and the Party's Central Committee, diabetes, along with cancer, hypertension, and cardiovascular diseases, are listed as the four major non-communicable diseases (NCDs) for which the goal is to "control the prevalence and reduce the probability of early death." ${ }^{26}$ Also, in 2017, the central government launched a 2017-2025 plan to control NCDs, which includes increasing the regular monitoring and selfmanagement rate for diabetes patients from 50\% (which is probably an overestimate) to $70 \%$ by $2025 .{ }^{27}$ To achieve population behavior change in any society is a difficult task; it is all the more so in large and diverse countries like China. Two national strategies, in particular, deserve special attention.

\section{National Demonstration Areas: A Short-term Strategy}

Since 2010, the Chinese Ministry of Health has established a network of $265 \mathrm{NDAs}$ to develop pilot projects for health promotion, detection and control of chronic diseases. To qualify for an NDA, county governments applied for central 
government subsidies and agreed to follow strict protocols. As of 2015, 9.29\% of China's counties established NDAs under the responsibility of China's Center for Disease Control (CDC). At the county level, various local government departments (propaganda, women's federation, education, health) are involved and in most cases the top county official is responsible for overall coordination. Primary care providers promote education and self-management of health conditions through appropriate dietary changes, control of tobacco use, and physical activity. ${ }^{28}$

A 2018 evaluation on the implementation of NDAs compared the 265 NDAs to one another along multiple criteria to assess their effectiveness in managing patients with diabetes and other NCDs. ${ }^{29}$ Aside from disparities across the NDAs, it found that health promotion activities and monitoring general population health were generally more successful than managing the conditions of those suffering from NCDs. With regard to diabetes, this conclusion most probably applies to all of China as it is consistent with the findings of Bragg and colleagues on the high proportion of deaths attributed to diabetic ketoacidosis or coma and chronic kidney disease mainly related to diabetes. ${ }^{24}$ These deaths are usually related to poor clinical management.

Another 2018 evaluation of the NDAs, specifically on the management of patients with diabetes, by Jin and colleagues, suggests that health promotion efforts resulting in lifestyle changes and self-management can nevertheless improve process measures of diabetes control. ${ }^{30}$ This study, conducted in 2016, on 3213 randomly selected adult residents (35 years and over) in NDAs, found that, among patients diagnosed with diabetes, $69.92 \%$ were assigned to a general practitioner (GP) and $53.66 \%$ reported receiving standardized management for their condition - at least one blood glucose test and four follow-up visits, per year, by a GP. It found that patients who are assigned to a GP, have a more positive attitude, and that patients without hypertension, are more likely to have their diabetes under better standardized control, ie, with patients receiving at least one blood glucose test and four follow-up visits, per year, by a GP. The study also found that patients in eastern provinces are under better standardized control, which could be the result of earlier establishment of NDAs; or alternatively, could be attributed to better health systems in these provinces. ${ }^{30}$ While the results of NDAs are encouraging, they also highlight the need for greater centralization of funding for public health interventions. The 2018 plan indicates that health spending has depended largely on local governments, which explains the wide disparities in access to health services among counties. ${ }^{31}$

Jin and colleagues conclude that "NDAs have achieved the goals of the 2012-2015 National NCD Control Plan, which requires a $40 \%$ standardized management rate." 30 They also find that $79.54 \%$ of patients in their study were aware of their diabetes diagnosis and $83.2 \%$ were in taking prescription drugs regularly to control their blood glucose levels. Both of these figures are high for China which suggests that NDAs have produced significant effects. It is difficult, however, to interpret these figures for several reasons. First, there were no control groups in this study - neither cross-sectional, nor before and after the intervention. They relied on comparisons with other national surveys that may not be comparable to theirs. Second, their survey question on self-reported high blood sugar levels is ambiguous. It is not sufficient to confirm the diagnosis and may include prediabetes patients. In fact, Jin and colleagues suggest that further surveys be conducted to see if standardized management has affected blood sugar control. Thus, we believe that this evaluation must be read with caution. Nonetheless, the NDAs represent important pilots for learning about disparities in prevalence, awareness and treatment of diabetes. Moreover, they are important for testing health promotion and monitoring tools, selfmanagement and standardized management approaches for controlling diabetes.

\section{Health System Integration: A Long-term Strategy}

Beyond the importance of pilot projects, the long-term strategy for confronting the diabetes challenge in China is to extend efforts at health promotion, detection and control of diabetes while simultaneously integrating academic medical centers (AMCs) and other hospitals with effective primary care. AMCs are overcrowded with patients who have simple conditions, while the primary care system is under-equipped with basic medical infrastructure and an under-qualified workforce. Since 2015, the State Council encouraged health system integration by promoting what the Chinese call a "hierarchical medical system,"32 a policy that attempts to attract more patients to primary health centers before going to AMCs.

The aspiration to achieve health system integration, in China, is as daunting as in any other health system. Moreover, China faces two significant obstacles. First, most primary care institutions are not qualified enough to earn patients' trust. In 2017, $82 \%$ of physicians and assistant physicians in rural township health centers and 55\% in urban community health centers have no bachelor's degree, in contrast to $70 \%$ of their counterparts in hospitals ${ }^{[1]} .^{2}$ In the absence of uniform professional standards, it is rational for patients to crowd into the best hospitals. Second, under the current system, hospitals have more abundant supplies of prescription drugs, partly because selling them is still an important part of hospital revenue. Although overcrowded, AMCs have little incentive to divert patients to primary care institutions.

In confronting these obstacles, municipal governments in big cities are attempting to integrate AMCs and community clinics into so-called medical partnerships. There are several models for achieving such health system integration. Some partnerships share only the same referral system; others have actually achieved deeper cooperation such that different institutions share the same workforce and operate under the same leadership. In general, cooperation is stronger in big cities than in rural areas. Shenzhen's Luohu model is an example of high-level of cooperation where primary care institutions and AMCs form a "hospital group" under the leadership of a common board of directors. Medical partnerships in big cities often resemble the Luohu model, though the level of cooperation varies. ${ }^{33}$ 


\section{The Future}

Although the implementation of NDAs and efforts to achieve health system integration are both well-conceived and necessary steps to confront the challenge of diabetes and more generally achieve China's goals for healthcare reform, these interventions have so far been inadequate. Two deeper long-term challenges remain before significant progress can be made in stemming the diabetes crisis: upgrade the primary care workforce; and reduce inequalities in access to healthcare.

\section{The Primary Care Workforce}

Long-term management of diabetes requires a competent primary care workforce backed up by available resources. However, these requirements are not yet met in China, neither for people with diabetes nor for the general population. Five percent of urban community health centers and $10 \%$ of township (rural) health centers are unable to provide basic services such as routine blood tests, urinalyses and electrocardiography. For village clinics, $96 \%$ are unable to provide routine blood tests, $90 \%$ are unable to provide urinalyses, and $31 \%$ are unable to provide blood glucose tests. Only $46 \%$ of patients with diabetes who usually seek care from primary healthcare institutions are diagnosed, and only $3 \%$ of them have their blood glucose controlled. Among all patients treated by primary care institutions, $84 \%$ are not satisfied and $39 \%$ of patients who bypassed primary care institutions noted their distrust as the reason for their choice. ${ }^{34}$

In 2016, the ratio of certified GPs per 10000 population was 1.6, far below high-income countries' average (Table 2). As China has sought to improve health system integration through the rapid expansion of the primary care workforce, the number of certified GPs has increased by $34 \%$ but the physician workforce in hospitals increased faster (by 57\%) than urban (15\%) and rural (37\%) primary care institutions. ${ }^{2}$

Unfortunately, GPs in China remind the population of barefoot doctors in the Mao era, during which professional standards of medicine were severely compromised to achieve universal coverage of basic necessities. Due to the transformation of China's healthcare system over the past decades, the term "doctor" refers to a wide range of professionals, from village doctors with months of training to graduates of the best medical schools. ${ }^{36}$ Although China is trying to reestablish professional standards through reforms in medical education, it will take time before the profession of GP becomes an attractive one for medical students and wins the trust of patients.

\section{Inequalities in Access to Healthcare}

Despite the proclaimed commitment to universal health coverage, as we noted earlier, the vast majority of China's migrants are covered by their home provinces, which provide them much less generous insurance coverage than their resident cities. Though efforts have been made to increase reimbursement in their resident cities, due to administrative barriers, it is still difficult for migrant workers to pay outof-pocket for healthcare and then wait to be reimbursed a small share of their outlays. ${ }^{37}$ This situation is most severe in large cities with the highest prevalence of migrants and
Table 2. Primary Care Workforce: China and Other Countries, 2016

\begin{tabular}{lcc}
\hline & GPs (Per 1000 Population) & Nurses (Per 1000 Population) \\
\hline China & 0.16 & 2.32 \\
Korea & 0.13 & 6.80 \\
Japan & - & 11.34 \\
US & 0.31 & 11.61 \\
Australia & 1.17 & 11.57 \\
UK & 0.76 & 7.88 \\
Canada & 1.27 & 9.91 \\
France & 0.90 & 10.21 \\
Germany & 0.70 & 12.84 \\
\hline
\end{tabular}

Abbreviation: GPs, general practitioner.

Source: National Commission of Health and Family Planning Statistical Yearbook $^{3}$ and OECD data. ${ }^{35}$

diabetes. For example, almost a third of Beijing's 21.71 million residents (8.08 million) do not have an urban residence card (hukou). ${ }^{38}$ The same situation prevails in Shanghai where 9.7 million residents, of its 24.2 million inhabitants, have no urban residence card. ${ }^{39}$

In these wealthy cities, since migrants are, on average, living in more precarious conditions, less educated, and less attentive to potential health problems, they tend to have higher than average rates of overweight and obesity and alcohol consumption. Their risk of diabetes is therefore higher than that of their official urban counterparts. ${ }^{22}$ Despite these wellrecognized problems, the Beijing and Shanghai 2030 Health Plans do not include specific measures for diabetes control among migrants. ${ }^{40,41}$

\section{Artificial Intelligence and Big Data}

Beyond the importance of taking action on health promotion strategies to improve diet, encourage exercise and increase greater awareness of risk factors for diabetes, China's health policy-makers have not overlooked significant advances in AI and Big Data. ${ }^{42}$ In some respects, the future is already here with respect to automated retinal screening (detection of diabetic retinopathy), clinical decision support (detection and monitoring of diabetes and comorbidities), predictive population risk stratification (identification of diabetes subpopulations at higher risk of complications) and patient self-management tools (eg, activity and dietary tracking devices and AI-improved glucose sensors). As these tools are improved, they will soon enable health professionals in China to identify some prediabetic patients and to diagnose complications related to diabetes such as retinopathy and renal diseases. ${ }^{43}$ On-going research on specific algorithms may also eventually allow more personalized targeting of diabetes treatment. ${ }^{44}$

Today, diabetes patients are generally more "connected" than patients with other chronic conditions. Activity trackers and smartphone applications have the potential to prevent type 2 diabetes by promoting the adoption of behaviors that prevent the onset of the disease, such as physical activity and dietary changes. Also, use of connected sensors or mobile applications already allow better daily monitoring of patients with type 2 diabetes. ${ }^{45}$ In addition to introducing innovations 
such as intelligent sensors for measuring and treating blood glucose levels, Ali Health, the medical branch of the giant Alibaba launched an AI physician system, Dia Doc, in May 2018. This system, dedicated to diabetes, brings together experienced specialists and practitioners, and guides GPs less familiar with the disease in performing diagnosis and treatment. ${ }^{46}$

China's digital transformation and its massive amounts of accumulated data provide an opportunity for the health system to leverage AI capabilities in improving diabetes care. ${ }^{47}$ Diet and exercise, two fundamental risk factors for most NCDs, are under the surveillance of Chinese tech-giants. E'leme (Are You Hungry?), a takeaway app recently acquired by the top tech-giant, Alibaba, has 260 million users, and Ali has already been collecting data on its users' diets for years. ${ }^{48}$ Ali's competitor Tencent, whose most successful app, WeChat has 1.08 billion users who use it at least once a month, ${ }^{49}$ has been collecting data on the average number of steps, per day, walked by the Chinese. ${ }^{50}$

Drawing on their subsidiary payment systems, Alipay and WeChat Pay, both Ali and Tencent are capable of analyzing nearly all aspects of the population's economic activities. Their ambition clearly extends beyond expanding membership, for health improvement strategies present a most desirable new frontier, especially since the government is encouraging the use of Big Data in the Healthy China 2030 development plan. ${ }^{26}$ Health was one of the top priorities in the government's plan to promote the use of Big Data and encourage cooperation among technology enterprises as a way to confront diabetes and other NCDs. ${ }^{51}$ Premier Li Keqiang, himself, has flagged Big Data as an area from which the public health sector should learn..$^{52}$

\section{Concluding Observations}

Faced with the world's largest population of people with diabetes that continues to grow with China's rapid urbanization, China has significantly improved the detection and management of diabetes, with its pilot projects in NDAs and its attempts to improve health system integration. These initiatives demonstrate a willingness to confront the rise of diabetes with strategies based on health promotion, disease detection and management of the multiple conditions and complications associated with the disease. AI and Big Data are likely to improve diagnosis and treatment. However, there is no nation in which the challenge of diabetes is likely to be solved by a technological fix. AI and Big Data must be financed and embedded in an institutional health system. Based on the literature and data we have reviewed and analyzed in this paper, significant challenges remain.

The extension to the rest of China of lessons learned from short-term strategies implemented in NDAs will require overcoming the fragmentation of a health system that still places excessive reliance on local government financing. Ongoing reform of the health system will require greater efforts to train and pay primary care providers to relieve a system that is too focused on large hospitals, and to achieve broader health system integration. Above all, longer-term success in meeting the challenge of diabetes will have to focus on upgrading the primary care workforce and reducing inequalities in access to healthcare.

\section{Acknowledgements}

We thank Manuel Raices Perez-Castañeda for his invitation to present a preliminary version of this paper to the International Diabetes Congress (IDC) in Varadero, Cuba in December, 2018. We are also grateful to Dr. Zhicheng Wang, School of Public Health, Peking University, Beijing, China, who provided useful information as we began our research. Finally, we thank our anonymous reviewers for most helpful suggestions and Wagner/NYU, New York City, NY, USA and University Paul Valéry-Montpellier 3 (IRIEC), Montpellier, France for travel support to attend the IDC.

\section{Ethical issues}

Not applicable.

Competing interests

Authors declare that they have no competing interests.

\section{Authors' contributions}

ZL and GF conducted the main documentary research for this paper within the overall framework developed by VGR. All three authors participated in drafting the paper and revising it for final submission.

\section{Authors' affiliations}

${ }^{1}$ Robert F. Wagner School of Public Service, New York University (NYU), New York City, NY, USA. ${ }^{2}$ IRIEC EA 740, Université Paul Valéry, Montpellier3, Montpellier, France. ${ }^{3}$ World Cities Project, Wagner School of Public Service, New York University (NYU), New York City, NY, USA.

\section{Endnote}

${ }^{[1]}$ In China, healthcare institutions are categorized as hospitals, urban community health centers and rural township health centers. Urban community and rural township health centers are considered as primary care institutions, but they also provide inpatient care. The yearbook does not provide strict and clear definitions but it is generally believed that hospitals provide comprehensive care while urban and rural health centers are smaller than hospitals and provide mainly primary care.

\section{References}

1. International Diabetes Federation. IDF Diabetes Atlas. 8th ed. https://diabetesatlas.org/resources/2017-atlas.html. Published 2017.

2. National Commission of Health. Chinese National Statistical Yearbook of Health. China, Beijing: Peking Union Medical College Press; 2018.

3. National Commission of Health and Family Planning. Chinese National Statistical Yearbook of Health and Family Planning. China, Beijing: Peking Union Medical College Press; 2017.

4. Holmes D. Linong Ji: Fighting to turn the tide against diabetes in China. Lancet. 2014;383(9933):1961. doi:10.1016/S01406736(14)60942-9

5. Pan X-R, Yang W-Y, Li G-W, Liu J. Prevalence of diabetes and its risk factors in China, 1994. Diabetes Care. 1997;20(11):1664-1669. doi:10.2337/diacare.20.11.1664

6. $\mathrm{Xu} \mathrm{Y}$, Wang $\mathrm{L}, \mathrm{He} \mathrm{J}$, et al. Prevalence and control of diabetes in Chinese adults. JAMA. 2013;310(9):948-959. doi:10.1001/ jama.2013.168118

7. Wang L, Gao P, Zhang $\mathrm{M}$, et al. Prevalence and ethnic pattern of diabetes and prediabetes in China in 2013. JAMA. 2017;317(24):2515-2523. doi:10.1001/jama.2017.7596

8. Yang W, Lu J, Weng J, et al. Prevalence of diabetes among men and women in China. N Engl J Med. 2010;362(12):1090-1101. doi:10.1056/NEJMoa0908292 
9. Hu D, Sun L, Fu P, et al. Prevalence and risk factors for type 2 diabetes mellitus in the Chinese adult population: the InterASIA Study. Diabetes Res Clin :Pract. 2009;84(3):288-295. doi:10.1016/j. diabres.2009.02.021

10. Popkin BM. Will China's nutrition transition overwhelm its health care system and slow economic growth? Health Aff (Millwood). 2008;27(4):1064-1076. doi:10.1377/hlthaff.27.4.1064

11. Hu C, Jia W. Diabetes in China: epidemiology and genetic risk factors and their clinical utility in personalized medication. Diabetes. 2018;67(1):3-11. doi:10.2337/dbi17-0013

12. $\mathrm{Li} \mathrm{Y,} \mathrm{He} \mathrm{Y,} \mathrm{Qi} \mathrm{L,} \mathrm{et} \mathrm{al.} \mathrm{Exposure} \mathrm{to} \mathrm{the} \mathrm{Chinese} \mathrm{famine} \mathrm{in} \mathrm{early} \mathrm{life}$ and the risk of hyperglycemia and type 2 diabetes in adulthood. Diabetes. 2010;59(10):2400-2406. doi:10.2337/db10-0385

13. van Abeelen AF, Elias SG, Bossuyt PM, et al. Famine exposure in the young and the risk of type 2 diabetes in adulthood. Diabetes. 2012;61(9):2255-2260. doi:10.2337/db11-1559

14. Fuentes S, Fosse-Edorh S, Regnault N, Goldberg M, Cosson E. Prevalence of Prediabetes and Undiagnosed Diabetes among Adults Aged 18 to 70 Years in France-The CONSTANCES Cohort. Diabetes 2018; 67(suppl 1). doi:10.2337/db18-1657-P

15. National Center for Chronic Disease Prevention and Health Promotion. National Diabetes Statistics Report, 2017. Estimates of Diabetes and Its Burden in the United States. https://www.cdc.gov/ diabetes/data/statistics/statistics-report.html. Published 2017.

16. Diabetes UK. Facts and Statistics. https://diabetes-resourcesproduction.s3-eu-west-1.amazonaws.com/diabetes-storage/ migration/pdf/Diabetes-key-stats-guidelines-April2014.pdf. Published 2014.

17. Sainsbury E, Shi Y, Falck J, Colagiuri S. Burden of Diabetes in Australia: Its Time for More Action Report. http://bit.do/faM3Q. Published 2018

18. World Health Organization. World Health Day 2016: Diabetes. http:// www.searo.who.int/india/mediacentre/events/2016/en/. Published 2016.

19. National Bureau of Statistics. The growth of per capita income and consumption in 2016. http://www.stats.gov.cn/tjsj/sjjd/201701/ t20170120_1456174.html. Published 2017.

20. Zhou M, Astell-Burt T, Bi Y, et al. Geographical variation in diabetes prevalence and detection in China: multilevel spatial analysis of 98,058 adults. Diabetes Care. 2015;38(1):72-81. doi:10.2337/dc141100

21. People's Daily. The number of migrant workers is declining. http:// society.people.com.cn/n1/2017/1115/c1008-29646364.html. Published 2017.

22. Bi $Y$, Wang $L, X u Y$, et al. Diabetes-related metabolic risk factors in internal migrant workers in China: a national surveillance study. Lancet Diabetes Endocrinol. 2016;4(2):125-135. doi:10.1016/ S2213-8587(15)00366-6

23. Su X. Migrant workers' utilization of health services: Shanghai as an example. School of Social Development and Public Policy, Fudan University; 2012.

24. Bragg F, Holmes MV, lona A, et al. Association between diabetes and cause-specific mortality in rural and urban areas of China. JAMA. 2017;317(3):280-289.

25. Chan M. China's burgeoning epidemic of diabetes-associated mortality. JAMA. 2017;317(3):264-266.

26. CCP Central Committee and State Council. Healthy China 2030 Plan. http://www.gov.cn/zhengce/2016-10/25/content_5124174. htm. Published 2016.

27. State Council. The National Long-term Plan to Control and Prevent Chronic Diseases, 2017-2025. http://www.gov.cn/zhengce/ content/2017-02/14/content_5167886.htm. Published 2017.

28. National Commission of Health and Family Planning. Achievements of National Demonstration Areas. http://www.gov.cn/xinwen/2016-11/20/ content_5135074.htm. Published 2016.

29. Li J, Li J, Zhang J, et al. Study on the overall implementation status of the National Demonstration Areas for Comprehensive Prevention and Control of Non-communicable Diseases. Zhonghua Liu Xing Bing Xue Za Zhi. 2018;39 (4):417-421. doi:10.3760/cma.j.i ssn.0254-6450.2018.04.006

30. Jin R, Li J, Zhang J, et al. Management programs on diabetes among Chinese adults in the National Demonstration Areas for Comprehensive Prevention and Control of Non-communicable Diseases. Zhonghua Liu Xing Bing Xue Za Zhi. 2018;39(4):407-411. doi:10.3760/cma.j.issn.0254-6450.2018.04.004

31. State Council. Health expenditure responsbilities of central and provincial governments. http://www.gov.cn/zhengce/content/2018-08/13/content 5313489.htm. Published 2018.

32. State Council. Promoting hierarchical medical system. http://www. gov.cn/zhengce/content/2015-09/11/content_10158.htm. Published 2015.

33. Li B. Director of National Committee of Health and Family Planning, Li Bin: Four Models of Medical Partnerships. http://www.sohu. com/a/128570598_452205. Published 2017.

34. LiX, Lu J, Hu S, et al. The primary health-care system in China. Lancet. 2017;390(10112):2584-2594. doi:10.1016/S0140-6736(17)33109-4

35. OECD. Health care resources, OECD Health Statistics (database). Primary care and nurse workforce. doi:10.1787/data-00541-en. Published 2016.

36. Zhu J, Li W, Chen L. Doctors in China: improving quality through modernisation of residency education. Lancet. 2016;388(10054):1922-1929. doi:10.1016/S0140-6736(16)00582-1

37. People's Daily. Further reform is required to make reimbursement more convenient for migrant workers. http://opinion.people.com.cn/ n1/2018/0302/c1003-29842690.htm. Published 2017.

38. Beijing Municipal Government. Beijing Statistical Yearbook 2018. http://tjj.beijing.gov.cn/nj/main/2018-tjnj/zk/indexch.htm. Published 2018.

39. Shanghai Municipal Government. Shanghai Statistical Yearbook 2018. http://www.stats-sh.gov.cn/tjnj/nj18.htm?d1=2018tjnj/C0201. htm. Published 2018

40. Beijing CCP Committee and Municipal Government. Healthy Beijing 2030. http://www.beijing.gov.cn/zfxxgk/110088/zzqgh33/2017-09/14/ content_5a0a51794dc347028b7069657d407c7c.shtml. Published 2007.

41. Shanghai CCP Committee Municipal Government. Healthy Shanghai 2030. http://wap.sh.gov.cn/nw2/nw2314/nw2319/nw12344/ u26aw55477.html. Published 2018.

42. Dankwa-Mullan I, Rivo M, Sepulveda M, Park Y, Snowdon J, Rhee K. Transforming diabetes care through artificial intelligence: the future is here. Popul Health Manag. 2019;22(3):229-242. doi:10.1089/ pop.2018.0129

43. Wee S-L, Mozur P. Amazon wants to disrupt health care in America. In China, tech giants already have. New York Times 31 January, 2018. https://www.nytimes.com/2018/01/31/technology/amazonchina-health-care-ai.html.

44. This how artificial intelligence is transforming diabetes care management. CB Insight. October 3, 2018. https://www.cbinsights. com/research/artificial-intelligence-diabetes-diagnosis-treatment/.

45. Fagherazzi G, Ravaud P. Digital diabetes: Perspectives for diabetes prevention, management and research. Diabetes Metab. 2019;45(4):322-329. doi:10.1016/j.diabet.2018.08.012

46. Ni D. Ali Health developed the first diabetes Al physician. 2018; http://company.cnstock.com/company/scp_gsxw/201805/4220563. htm.

47. Fabre G. China's digital transformation. Why is artificial intelligence a priority for chinese R\&D? https://halshs.archives-ouvertes.fr/ halshs-01818508v2/document. Published 2018.

48. Xinhua News. Ali wholly-owned acquired E'leme with 9.5 billion US dollars. http://www.xinhuanet.com/tech/2018-04/03/c_1122629159. htm. Published 2018.

49. Tencent. WeChat Data Report, 2018. https://support.weixin.qq.com/ cgi-bin/mmsupport-bin/getopendays. Published 2018.

50. China Daily. QQ Big Data: How many steps an average Chinese walks per day? http://tech.chinadaily.com.cn/a/201901/08/ WS5c3409caa3100a343d6f28d0.html. Published 2019.

51. State Council. China's plan to promote the development of Big Data. http://www.gov.cn/zhengce/content/2015-09/05/content_10137. htm. Published 2015.

52. State Council. Li Keqiang: Health Big Data should focus on urgent demands of the people. http://www.gov.cn/guowuyuan/2016-06/08/ content_5080599.htm. Published 2016. 